\title{
Split-Plot Central Composite Designs Robust to a Pair of Missing Observations
}

\author{
${ }^{1}$ YAKUBU, Y; ${ }^{2}$ CHUKWU, A U \\ ${ }^{I}$ Department of Statistics, Federal University of Technology, Minna, Niger State, Nigeria. \\ ${ }^{2}$ Department of Statistics, University of Ibadan, Ibadan, Oyo state, Nigeria. Email: Unnachuks2002@yahoo.co.uk \\ "Corresponding Author Email: yisa_yakubu@yahoo.com
}

\begin{abstract}
This study constructs robust split-plot central composite designs against missing pairs of observations. Split-plot central composite designs (CCD) consist of factorial $(f)$, whole-plot axial $(\alpha)$, subplot axial $(\beta)$, and center $(c)$ points. A loss function in terms of determinant $(D)$ criterion was formulated based on two different configurations of the factorial and axial parts; losses due to missing pairs of observations of these different categories of points were investigated. Robust split-plot central composite designs against missing pairs of observations were then developed under each of the two configurations. It was observed that the losses, $L_{f f}, L_{\beta \beta}$, and $L_{f \beta}$, due respectively, to missing pairs of observations of the factorial, subplot axial, and, factorial and subplot axial points, were higher than the losses due to missing pairs of observations of the whole-plot axial and center points given by $L_{\alpha \alpha}$ and $L_{c c}$ respectively. Thus the factorial $(f)$ and the subplot axial $(\beta)$ points were found to be the most influential points in these designs while the whole-plot axial $(\alpha)$ and the center $(c)$ points were less influential. This work has therefore identified and properly classified the losses due to missing design points in the split-plot CCD portions. In this way, the practitioner can avoid the experimental points having less influence from the full CCD experiments and this could lead to a possible increase in design efficiency.
\end{abstract}

\section{DOI: https://dx.doi.org/10.4314/jasem.v22i9.08}

Copyright: Copyright $\odot 2018$ Yakubu and Chukwu. This is an open access article distributed under the Creative Commons Attribution License (CCL), which permits unrestricted use, distribution, and reproduction in any medium, provided the original work is properly cited.

Dates: Received: 04 June 2018; Revised: 01August 2018; Accepted: 16 August 2018

Keywords: Robustness, Split-plot Central Composite Designs, Missing observations, loss function

Response Surface Methodology (RSM) is an area of experimental design which consists of a group of mathematical and statistical techniques used in the development of an adequate functional relationship between a response of interest, $\mathrm{y}$, and a number of associated control (or input) variables denoted by $x_{1}, x_{2}, \ldots, x_{k}$ (Myers et al, 2009). RSM has continued to play vital roles in developing, optimizing and improving processes, particularly where several input variables, $x_{1}, x_{2}, \ldots, x_{k}$, potentially influence some performance measure or quality characteristic, $y$, of the process under study. However, one difficulty in applying classical response surface designs is that they inherently assume that all factors are equally easy to manipulate, thereby allowing for complete randomization of experimental run order. In practice, most industrial experiments cannot be completely randomized due to the presence of some factors with hard-to-change (HTC) levels. Therefore, these experiments are often conducted in a manner that restricts the randomization, which leads to a split-plot structure. In a split-plot design, experimental runs are performed in groups, where in a group, the levels of the HTC factors are not reset from run to run. This creates dependence among the runs in one group, and leads to clusters of correlated errors and responses. The first paper to exclusively focus on conducting response surface experiments with a split-plot structure was by Myers and Lentner (Letsinger et al, 1996). The authors investigate efficiency of various second-order response surface designs when conducted with a split-plot structure. This was then followed by other authors like Vining et al (2005), Kowalski et al (2006) etc., who modified completelyrandomized central composite and Box-Behnken designs (CCD and BBD) to accommodate a split-plot structure. Split-plot central composite designs (CCD) consist of factorial $(f)$, whole-plot axial $(\alpha)$, subplot axial $(\beta)$, and center $(c)$ points.

Even in a carefully-planned experiments, situations of missing observations cannot be avoided due to reasons that are beyond an experimenter's control. When some observations are lost, desirable design properties such as independence, optimality and orthogonality etc., are destroyed. In this situation, parameters of the fitted model cannot be separately estimated and the design may break down. Thus there is strong need for designs that are insensitive or robust to missing observations. A number of researchers have studied the loss due to a set of missing observations over a range of $\alpha$ value associated with completely randomized central composite designs (CCD) using the loss function introduced by Akhtar and Prescott (1986). Since then, minimaxloss designs that are robust to sets of missing observations have effectively emerged. Much of these studies consider the second-order central composite designs differing in number of control variables and configurations of the factorial, axial and center portions. Some of such results include Akhtar and 
Prescott (1986), Akhtar (2001) and Akram (2002). However, little or no work has been done on investigating the loss due to missing observations in response surface designs conducted within a split-plot structure. Therefore, the objective of this study is to assess the loss due to missing pairs of observations in split-plot central composite designs and to construct split-plot central composite designs that are robust against these missing pairs of observations.

\section{MATERIALS AND METHODS}

Candidate Split-plot Designs: Two different configurations of split-plot central composite designs (CCDs) were considered. These include:

Split-plot CCDs with single replication of factorial and axial parts $(\mathrm{F}+\mathrm{A})$.

Split-plot CCDs with half replicate of factorial part and complete replicate of axial part (1/2 F+A).

For each of these configurations, five (5) different candidate split-plot CCDs with factors between 3 and 6 were selected to establish the validity of the criterion. These designs are given in the table below with $k, w$ and $s$ denoting, respectively, numbers of design variables, whole-plot variables and subplot variables.

\begin{tabular}{cccccc}
\multicolumn{3}{c}{ Table.1: Candidate Split-plot CCDs } \\
\hline \multicolumn{3}{c}{$(\mathrm{F}+\mathrm{A})$} & \multicolumn{3}{c}{$(1 / 2 \mathrm{~F}+\mathrm{A})$} \\
\hline $\boldsymbol{k}$ & $w$ & $s$ & $\boldsymbol{k}$ & $w$ & $s$ \\
$\mathbf{4}$ & 1 & 3 & $\mathbf{5}$ & 1 & 4 \\
$\mathbf{4}$ & 2 & 2 & $\mathbf{5}$ & 2 & 3 \\
$\mathbf{5}$ & 2 & 3 & $\mathbf{6}$ & 2 & 4 \\
$\mathbf{5}$ & 3 & 2 & $\mathbf{6}$ & 3 & 3 \\
& & & $\mathbf{6}$ & 1 & 5 \\
\hline
\end{tabular}

Now, for a k-factor $\mathrm{D}(w, s)$ split-plot CCD with four categories of design points, there are ten possible pairs of missing observations from factorial $(f)$, whole-plot axial $(\alpha)$, subplot axial $(\beta)$, and center $(c)$ points. These include $f f, \alpha \alpha, \beta \beta, c c, f \alpha, f \beta, f c, \alpha \beta, \alpha c$, and $\beta c$. In terms of the derived Minimaxloss criterion, our computations show that the loss in design information due to missing any of the pairs: $\alpha \alpha, c c, f \alpha, f c, \alpha \beta, \alpha c$ and $\beta c$, denoted respectively by $L_{\alpha \alpha}, L_{c c}, L_{f \alpha}, L_{f c}, L_{\alpha \beta}$, $L_{\alpha c}$, and $L_{\beta c}$, did not have as much adverse effect on the design as that due to missing $f f, \beta \beta$, and $f \beta$. Therefore, we investigate the robustness of these designs in the presence of the missing pairs: $c c, \alpha \alpha, f f, \beta \beta, f \beta$, and construct robust designs accordingly.

Loss function with respect to the Determinant Criterion (minimaxloss criterion): For split-plot response surface designs, the generalized least squares (GLS) model is

$$
\boldsymbol{y}=X \boldsymbol{\beta}+Z \gamma+\varepsilon
$$

Where $\boldsymbol{y}$ is the $N x 1$ vector of responses, $X$ is the $N x$ $p$ model matrix, $\beta$ is the $p \times 1$ vector of coefficients, $Z$ is an $N x b$ incidence matrix assigning observations to each of the $b$ whole plots; $\gamma$ is the $N \times 1$ vector of random whole-plot errors, $\varepsilon$ is the $N \times 1$ vector of random subplot errors. It is assumed that $\gamma_{i} \sim N\left(0, \sigma_{\gamma}^{2}\right), \quad \varepsilon_{i j} \sim N\left(0, \sigma_{\varepsilon}^{2}\right), \operatorname{cov}\left(\gamma_{i}, \varepsilon_{i j}\right)=0$.

The variance - covariance matrix for the observation vector $\mathbf{y}$ is

$$
\begin{gathered}
\operatorname{Var}(y)=V=\sigma_{\varepsilon}^{2} I_{n}+\sigma_{\gamma}^{2} Z Z^{\prime} \\
=\sigma_{\varepsilon}^{2}\left(I_{n}+d Z Z^{\prime}\right)
\end{gathered}
$$

where $d=\frac{\sigma_{\gamma}^{2}}{\sigma_{\varepsilon}^{2}} ; \sigma_{\gamma}^{2}$ and $\sigma_{\varepsilon}^{2}$ are the whole-plot and subplot error variances respectively.

The matrix $\boldsymbol{Z Z}$ ' is a block diagonal matrix with diagonal matrices of ${ }_{1}, J_{n 2}, \ldots, J_{n z}$, where $J_{n i}$ is an $n_{i} \mathrm{X}$ $n_{i}$ matrix of 1's.

The GLS estimates are

$$
\begin{aligned}
\hat{\beta}_{G L S}= & \left(X^{\prime} V^{-1} X\right)^{-1} X^{\prime} V^{-1} y \\
& \hat{y}=X\left(X^{\prime} V^{-1} X\right)^{-1} X^{\prime} V^{-1} y=H y
\end{aligned}
$$

$$
\begin{aligned}
& \operatorname{Var}\left(\underline{\hat{\beta}}_{G L S}\right) \\
& =\left(X^{\prime} V^{-1} X\right)^{-1} X^{\prime} V^{-1} \operatorname{Var}(\underline{y}) V^{-1} X\left(X^{\prime} V^{-1} X\right)^{-1} \\
& =\left(X^{\prime} V^{-1} X\right)^{-1} \quad, \text { since } \operatorname{Var}(\underline{y})=V
\end{aligned}
$$

Where $\boldsymbol{X}$ is as stated above and $\mathbf{y}$ is the vector of responses.

Following Akhtar and Prescott (1986) criterion for completely randomized central composite designs, Angela et al (2013) defined the minimaxloss criterion due to a single missing design point $u$ in a split-plot central composite design as

$$
\begin{aligned}
l_{u} & =\frac{\left|X^{\prime} V^{-1} X\right|-\left|X^{\prime} V^{-1} X\right|_{u}}{\left|X^{\prime} V^{-1} X\right|} \\
& =1-\frac{\left|X^{\prime} V^{-1} X\right|_{u}}{\left|X^{\prime} V^{-1} X\right|}, \quad u=1,2, \ldots \text { (4) }
\end{aligned}
$$

Where $\left|X^{\prime} V^{-1} X\right|_{u}$ is the determinant of the reduced information matrix due to the $\mathrm{u}^{\text {th }}$ missing point. $l_{u}$ is the loss and lies between zero and one. While the authors use this criterion to develop robust split-plot CCDs in the presence of a single missing observation, this present work develops split-plot CCDs that are robust to missing pairs ( $u v)$ of observations using

$$
\begin{gathered}
l_{u v}=\frac{\left|X^{\prime} V^{-1} X\right|-\left|X^{\prime} V^{-1} X\right|_{u v}}{\left|X^{\prime} V^{-1} X\right|} \\
=1-\frac{\left|X^{\prime} V^{-1} X\right|_{u v}}{\left|X^{\prime} V^{-1} X\right|}, \quad u, v=1,2, \ldots
\end{gathered}
$$

The missing observations are at the subplot level, and the whole -plot axial point distance equals the subplot axial point distance from the design center (i.e., $\alpha=\beta$ ).

\section{RESULTS AND DISCUSSIONS}

The computed maximum losses $\left(L_{f f}, L_{\alpha \alpha}, L_{\beta \beta}, L_{c c}\right.$, and $L_{f \beta}$ ) due to missing pairs of observations of factorial $(f)$, whole-plot axial $(\alpha)$, subplot $\operatorname{axial}(\beta)$, and center $(c)$ points are presented in the tables below for each of the selected split-plot CCDs and under each of 
the two configurations. The robust (or minimaxloss) designs are denoted by asterisks with the respective parameters as shown in each of the tables. These are the designs with the smallest maximum loss among all the maximum losses. We first consider the designs with single replication of factorial and axial parts $(\mathrm{F}+\mathrm{A})$. Under this configuration, the losses corresponding to missing pairs of observations are given respectively in TABLES $2-5$ below for the split-plot CCDs: $\mathrm{D}(1,3), \mathrm{D}(2,2), \mathrm{D}(3,2)$, and $\mathrm{D}(2,3)$. For each of these CCDs, the losses were also plotted against their corresponding $\alpha$ values and the loss curves given respectively as items $a, b, c$, and $d$, in FIGURE 1.

Comparing the different losses, it can be seen from each of the tables that there is a point $(\alpha)$ where the values of $L_{f f}$ and $L_{\beta \beta}$ coincide, as can also be seen from the corresponding figures. That is, at this point, the maximum loss is minimized. Thus our formulated loss function for split-plot CCDs performs as expected and compared favorably with that of Akhtar and Prescott, (1986) for completely randomized CCDs. From TABLE 2, we see that the four-factor split-plot CCD with $w=1, s=3, n_{f}=16, n_{\alpha}=16, n_{\beta}=$ 6, $n_{c}=8$ and $\alpha=\beta=2.059$ is a minimaxloss design robust to a pair of missing observations.

From TABLE 3, we can see that the four-factor splitplot CCD with $w=2, s=2, n_{f}=16, n_{\alpha}=16$, $n_{\beta}=4, n_{c}=4$ and $\alpha=\beta=2.0933 \quad$ is a minimaxloss design robust to a pair of missing observations. From TABLE 4, we observed that the values of $L_{f f}$ and $L_{\beta \beta}$ coincide at the point $\alpha=\beta=$ 1.6042 for the $\mathrm{D}(3,2)$ design. Thus the five-factor split-plot CCD with $w=3, s=2, n_{f}=32, n_{\alpha}=$ $24, n_{\beta}=4, n_{c}=4$, and $\alpha=\beta=1.6042$ is a minimaxloss design robust to a pair of missing observations.

We can observe from TABLE 5 that for the $\mathrm{D}(2,3)$ $\mathrm{CCD}$, the values of $L_{f f}$ and $L_{\beta \beta}$ coincide at the point where $\alpha=\beta=1.339$. Thus, the five-factor split-plot CCD with $w=2, s=3, n_{f}=32, n_{\alpha}=32, n_{\beta}=$ $6, n_{c}=8$, and $\alpha=\beta=1.339$ is a minimaxloss design robust to a pair of missing observations.

These points of minimum loss can also be seen directly from items $a-d$ in FIGURE 1, where the loss curves $L_{f f}$ and $L_{\beta \beta}$ intersect at the given value $(\alpha=\beta)$. Similar plots were used in Akhtar (2001) for completely randomized CCD. We can see from items $b$ and $c$, that the loss curve, $L_{c c}$, makes a bell-shaped curve when plotted against $\alpha$, attaining it's maximum at $\alpha=\sqrt{k}$, while $L_{\alpha \alpha}$ increases gradually with increasing $\alpha$. Item $a$ in FIGURE 1 shows that $L_{c c}$ and $L_{\alpha \alpha}$ overlap and slightly make a bell-shaped curve when plotted against $\alpha$, while in item $d, L_{c c}$ and $L_{\alpha \alpha}$ overlap and continue to increase gradually as $\alpha$ increases.

Then the designs with half replicate of factorial part and complete replicate of axial part $(1 / 2 \mathrm{~F}+\mathrm{A})$ were considered. Under this configuration, the losses corresponding to missing a pair of observations of each of the different categories of design points are given respectively in TABLES $6-10$ for the split-plot designs $\mathrm{D}(1,4), \mathrm{D}(2,3), \mathrm{D}(2,4), \mathrm{D}(3,3)$, and $\mathrm{D}(1,5)$.

These losses were also plotted against the various $\alpha$ values and given respectively as items $a, b, c, d$, and $e$, in FIGURE 2. Our computations of losses have shown that, for each of these designs also, the losses corresponding to missing a pair of center points, $\left(L_{c c}\right)$, and those corresponding to missing a pair of axial points $\left(L_{\alpha \alpha}\right)$, were each less than $L_{f f}$ and $L_{\beta \beta}$ for the whole range of $\alpha$, and also that $L_{c c}$ continues to increase up to the point $\alpha=\sqrt{k}$, and then decreases as $\alpha$ increases beyond this point. It can also be seen from each of the tables that the losses $L_{f f}$ decreases gradually with increasing value of $\alpha$, whereas $L_{\beta \beta}$ has an increasing trend with increasing $\alpha$.

\begin{tabular}{|c|c|c|c|c|c|c|}
\hline 1.0 & 0.0190 & 0.8942 & 0.0263 & 0.7091 & 0.8026 & 0.8942 \\
\hline 1.20 & 0.0222 & 0.8851 & 0.0278 & 0.7335 & 0.8076 & 0.8851 \\
\hline 2.0 & 0.0357 & 0.8428 & 0.0316 & 0.8328 & 0.8363 & 0.8428 \\
\hline 2.059 & 0.0356 & $0.8397 * * *$ & 0.0318 & $0.8397 * * *$ & 0.8390 & $0.8397 * * *$ \\
\hline 2.2 & 0.0345 & 0.8325 & 0.0322 & 0.8554 & 0.8453 & 0.8554 \\
\hline 2.5 & 0.0304 & 0.8184 & 0.0329 & 0.8846 & 0.8587 & 0.8846 \\
\hline 3.0 & 0.0238 & 0.7981 & 0.0338 & 0.9205 & 0.8791 & 0.9205 \\
\hline
\end{tabular}


Table 3: Losses due to different pairs of missing observations for four-factor $\mathrm{D}(2,2)$ split-plot CCD with single replication of factorial and axial parts; $n_{f}=16, n_{\alpha}=16, n_{\beta}=4, n_{c}=4, N_{w}=10$, and $N=40$.

\begin{tabular}{cccccc}
\hline$\alpha$ & $\begin{array}{c}\text { maxloss due to } \\
\text { missing a pair of } \\
\text { center points }\left(L_{c c}\right)\end{array}$ & $\begin{array}{c}\text { maxloss due to } \\
\text { missing a pair of } \\
\text { factorial points } \\
\left(L_{f f}\right)\end{array}$ & $\begin{array}{c}\text { maxloss due to } \\
\text { missing a pair of } \\
\text { whole-plot axial } \\
\text { points }\left(L_{\alpha \alpha}\right)\end{array}$ & $\begin{array}{c}\text { maxloss due to } \\
\text { missing a pair of } \\
\text { subplot axial points } \\
\left(L_{\beta \beta}\right)\end{array}$ & $\begin{array}{c}\text { maxloss due to missing } \\
\text { one factorial and one } \\
\text { subplot axial points } \\
\left(L_{f \beta}\right)\end{array}$ \\
\hline 1.0 & 0.0614 & 0.8663 & 0.1081 & 0.6499 & 0.7651 \\
1.20 & 0.0761 & 0.8563 & 0.1147 & 0.6773 & 0.7712 \\
1.50 & 0.1119 & 0.8397 & 0.1229 & 0.7182 & 0.7815 \\
1.75 & 0.1488 & 0.8244 & 0.1286 & 0.7901 & 0.8048 \\
2.0 & 0.1666 & 0.8094 & 0.1342 & $0.8044 * *$ & 0.8592 \\
2.0933 & 0.1642 & $0.8044^{* * *}$ & 0.1363 & 0.8592 & 0.8359 \\
3.5 & 0.1241 & 0.7873 & 0.1506 & 0.9046 & 0.8623 \\
\hline
\end{tabular}

***Minimaxloss due to two missing observations.

Table 4: Losses due to different pairs of missing observations for five-factor $\mathrm{D}(3,2)$ split-plot CCD with single replication of factorial and axial parts; $n_{f}=32, n_{\alpha}=24, n_{\beta}=4, n_{c}=4, N_{w}=16$, and $N=64$.

\begin{tabular}{|c|c|c|c|c|c|c|}
\hline$\alpha$ & $\begin{array}{l}\text { maxloss due to } \\
\text { missing a pair of } \\
\text { center points }\left(L_{c c}\right)\end{array}$ & $\begin{array}{l}\text { maxloss due to } \\
\text { missing a pair of } \\
\text { factorial points } \\
\left(L_{f f}\right)\end{array}$ & $\begin{array}{l}\text { maxloss due to } \\
\text { missing a pair of } \\
\text { whole-plot axial } \\
\text { points }\left(L_{\alpha \alpha}\right)\end{array}$ & $\begin{array}{l}\text { maxloss due to } \\
\text { missing a pair of } \\
\text { subplot axial } \\
\text { points }\left(L_{\beta \beta}\right)\end{array}$ & $\begin{array}{l}\text { maxloss due to } \\
\text { missing one } \\
\text { factorial and one } \\
\text { subplot axial } \\
\text { points }\left(L_{f \beta}\right)\end{array}$ & $\begin{array}{l}\text { Overall maxloss } \\
\text { due to missing } \\
\text { pairs of } \\
\text { observations }\end{array}$ \\
\hline 1.0 & 0.0433 & 0.6846 & 0.0980 & 0.6164 & 0.6395 & 0.6846 \\
\hline 1.20 & 0.0520 & 0.6793 & 0.1031 & 0.6327 & 0.6459 & 0.6793 \\
\hline 1.50 & 0.0746 & 0.6706 & 0.1102 & 0.6584 & 0.6562 & 0.6706 \\
\hline 1.6042 & 0.0863 & $0.6673 * * *$ & 0.1125 & $0.6673 * * *$ & 0.6599 & $0.6673 * * *$ \\
\hline 1.75 & 0.1066 & 0.6626 & 0.1154 & 0.6796 & 0.6651 & 0.6796 \\
\hline 2.0 & 0.1470 & 0.6538 & 0.1200 & 0.7019 & 0.6750 & 0.7019 \\
\hline 2.236 & 0.1666 & 0.6456 & 0.1247 & 0.7286 & 0.6883 & 0.7286 \\
\hline 2.50 & 0.1448 & 0.6380 & 0.1306 & 0.7644 & 0.7083 & 0.7644 \\
\hline 3.0 & 0.0823 & 0.6272 & 0.1397 & 0.8225 & 0.7450 & 0.8225 \\
\hline
\end{tabular}

****Minimaxloss due to two missing observations.

Table 5: Losses due to different pairs of missing observations for five-factor $\mathrm{D}(2,3)$ split-plot CCD with single replicationof factorial and axial parts; $n_{f}=32, n_{\alpha}=32, n_{\beta}=6, n_{c}=8, N_{w}=10$, and $N=78$.

\begin{tabular}{|c|c|c|c|c|c|c|}
\hline$\alpha$ & $\begin{array}{l}\text { maxloss due to } \\
\text { missing a pair } \\
\text { of center points } \\
\left(L_{c c}\right)\end{array}$ & $\begin{array}{l}\text { maxloss due to } \\
\text { missing a pair of } \\
\text { factorial points } \\
\left(L_{f f}\right)\end{array}$ & $\begin{array}{l}\text { maxloss due to } \\
\text { missing a pair of } \\
\text { whole-plot axial } \\
\text { points }\left(L_{\alpha \alpha}\right)\end{array}$ & $\begin{array}{l}\text { maxloss due to } \\
\text { missing a pair of } \\
\text { subplot axial points } \\
\left(L_{\beta \beta}\right)\end{array}$ & $\begin{array}{l}\text { maxloss due to } \\
\text { missing one } \\
\text { factorial and one } \\
\text { subplot axial points } \\
\left(L_{f \beta}\right)\end{array}$ & $\begin{array}{l}\text { Overall maxloss } \\
\text { due to missing } \\
\text { pairs of } \\
\text { observations }\end{array}$ \\
\hline 1.0 & 0.0142 & 0.7115 & 0.0234 & 0.6789 & 0.6819 & 0.7115 \\
\hline 1.25 & 0.0171 & 0.7060 & 0.0252 & 0.6970 & 0.6894 & 0.7060 \\
\hline 1.339 & 0.0185 & $0.7039 * * *$ & 0.0258 & $0.7039 * * *$ & 0.6923 & $0.7039 * * *$ \\
\hline 1.50 & 0.0216 & 0.6999 & 0.0268 & 0.7167 & 0.6979 & 0.7167 \\
\hline 1.75 & 0.0275 & 0.6933 & 0.0281 & 0.7375 & 0.7075 & 0.7375 \\
\hline 2.0 & 0.0333 & 0.6862 & 0.0291 & 0.7597 & 0.7185 & 0.7597 \\
\hline 2.236 & 0.0357 & 0.6795 & 0.0300 & 0.7825 & 0.7307 & 0.7825 \\
\hline 2.5 & 0.0331 & 0.6725 & 0.0309 & 0.8086 & 0.7459 & 0.8086 \\
\hline
\end{tabular}

***Minimaxloss due to two missing observations.

Among all the possible groups of pairs within every factorial whole plot for the $\mathrm{D}(1,4) \mathrm{CCD}$, we observed that ff1, ff3, and ff5 were empty, i.e., they don't exist, and only ff2 and ff4 were non-empty. Our computations of losses have shown that

$L_{f f 4}>L_{f f 2} ; \quad L_{\alpha \alpha 0}>L_{\alpha \alpha 1} ;$ and $L_{f \beta 1}>L_{f \beta 2}$.

Minimaxloss designs were therefore developed for the groups with maximum losses. From the losses in TABLE 6 for the D $(1,4)$ design, we observed that the maximum loss $\left(L_{\beta \beta}=0.9893\right)$ is minimum when $L_{f f}=L_{\beta \beta}=0.9893$ for $\alpha=\beta=5.995$ as indicated in the last column of the table. Thus, the five-factor $\mathrm{D}(1,4)$ split-plot CCD with half replication of factorial part and complete replication of axial part, and with $n_{f}=16, n_{\alpha}=16, n_{\beta}=8, n_{c}=8$, and $\alpha=\beta=$
5.995 is a minimaxloss design robust to a pair of missing observations.

Among all the possible groups of pairs within every factorial whole plot for the $\mathrm{D}(2,3) \mathrm{CCD}$, we observed that ff1, ff3, and ff5 were empty. The only non-empty pairs were ff 2 and ff 4 . Our computations of losses have shown that

$L_{f f 4}>L_{f f 2} ; L_{\alpha \alpha 0}>L_{\alpha \alpha 1}>L_{\alpha \alpha 2} ;$ and $L_{f \beta 1}>L_{f \beta 2}$.

From TABLE 7, we observed that the maximum loss $\left(L_{\beta \beta}=0.9617\right)$ is minimum when $L_{f f}=L_{\beta \beta}=$ 0.9617 for $\alpha=\beta=4.043$. Thus, the five-factor $\mathrm{D}(2,3)$ split-plot CCD with half replication of factorial part and complete replication of axial part, and with $n_{f}=16, n_{\alpha}=16, n_{\beta}=6, n_{c}=4$, and $\alpha=\beta=$ 
4.043 is a minimaxloss design robust to a pair of missing observations.

Among all the possible groups of pairs within every factorial whole plot for the $\mathrm{D}(2,4) \mathrm{CCD}$, we observed that ff1, ff3, ff5, and ff6 were empty. The only nonempty pairs were ff 2 and ff4. Our computations of losses have shown that

$L_{f f 4}>L_{f f 2} ; \quad L_{\alpha \alpha 0}>L_{\alpha \alpha 1}>L_{\alpha \alpha 2} ;$ and $L_{f \beta 1}>L_{f \beta 2}$.

We can observe from TABLE 8 that the maximum loss $\left(L_{\beta \beta}=0.8394\right)$ is minimum when $L_{f f}=L_{\beta \beta}=$ 0.8394 for $\alpha=\beta=2.665$. Thus, the six-factor $\mathrm{D}(2,4)$ split-plot CCD with half replication of factorial part and complete replication of axial part and with $n_{f}=32, n_{\alpha}=32, n_{\beta}=8, n_{c}=8$, and $\alpha=\beta=$ 2.665 is a minimaxloss design robust to a pair of missing observations.

Among all the possible groups of pairs within every factorial whole plot for the $\mathrm{D}(3,3) \mathrm{CCD}$, we observed that ff1, ff 3 , ff4, ff5, and ff6 were all empty. The only non-empty pair was the ff 2 . Our computations of losses have shown that

$$
L_{\alpha \alpha 0}>L_{\alpha \alpha 1} ; L_{f \beta 1}>L_{f \beta 2} \text {. }
$$

We can observe from TABLE 9 that the maximum loss $\left(L_{\beta \beta}=0.8278\right)$ is minimum when $L_{f f}=L_{\beta \beta}=$ 0.8278 for $\alpha=\beta=2.739$. Thus, the six-factor $\mathrm{D}(3,3)$ split-plot CCD with half replication of factorial part and complete replication of axial part and with $n_{f}=32, n_{\alpha}=24, n_{\beta}=6, n_{c}=4$, and $\alpha=\beta=$ 2.739 is a minimaxloss design robust to a pair of missing observations. Among all the possible groups of pairs within every factorial whole plot for the $\mathrm{D}(1,5)$ $\mathrm{CCD}$, we observed that $\mathrm{ff} 1$, ff 3 , ff5, and ff6, and ff6 were all empty. The only non-empty pairs were ff 2 and ff4. Our computations of losses have shown that

$L_{f f 2}>L_{f f 4} ; L_{\alpha \alpha 0}>L_{\alpha \alpha 1}>L_{\alpha \alpha 2} ; L_{f \beta 1}>L_{f \beta 2}$.

We can observe from TABLE 10 that the maximum loss $\left(L_{\beta \beta}=0.8526\right)$ is minimum when $L_{f f}=L_{\beta \beta}=$ 0.8526 for $\alpha=\beta=2.706$. Thus, the six-factor
$\mathrm{D}(1,5)$ split-plot CCD with half replication of factorial part and complete replication of axial part and with $n_{f}=32, n_{\alpha}=32, n_{\beta}=10, n_{c}=16$, and $\alpha=$ $\beta=2.706$ is a minimaxloss design robust to a pair of missing observations.

We can see from item $a$ in FIGURE 2 that the curves $L_{c c}$ and $L_{\alpha \alpha}$ overlap each other up to the point $(2.5000<\alpha<3.000)$, and that the two curves each makes a bell-shape when plotted against $\alpha$. In items $b$ and $d, L_{c c}$ makes a bell-shaped curve when plotted against $\alpha$, while $L_{\alpha \alpha}$ increases sharply as $\alpha$ increases. In item $c, L_{c c}$ and $L_{\alpha \alpha}$ overlap each other and make a bell-shaped curve with the horizontal $(\alpha)$ axis. In item $e, L_{c c}$ and $L_{\alpha \alpha}$ overlap each other and maintain a parallel position with the horizontal $(\alpha)$ axis.
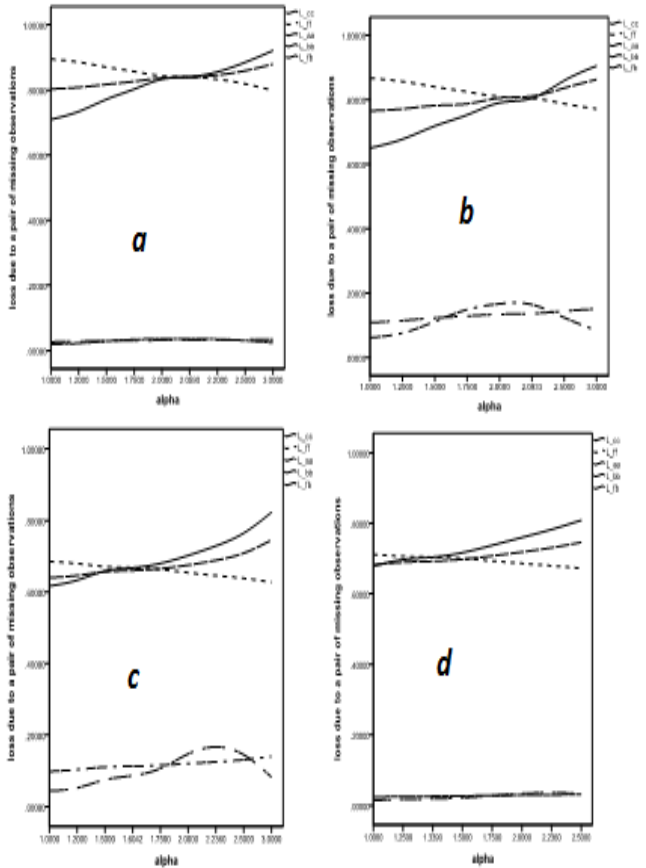

Figure 1: Loss curves due to a pair of missing observations for (a) $[\mathrm{D}(1,3)](\mathrm{b})[\mathrm{D}(2,2)]$ (c) $[\mathrm{D}(3,2)]$ and (d) $[\mathrm{D}(2,3)]$ with single replicate of factorial and axial points $(\mathrm{F}+\mathrm{A})$

Table 6: Losses due to different pairs of missing observations for five-factor $\mathrm{D}(1,4)$ split-plot CCD with half replication of factorial and complete replication of axial parts; $n_{f}=16$ from half replicate of factorial part with highest-order interaction as defining contrast, $n_{\alpha}=16, n_{\beta}$

\begin{tabular}{|c|c|c|c|c|c|c|}
\hline$\alpha$ & $\begin{array}{l}\text { maxloss due to } \\
\text { missing a pair } \\
\text { of center points } \\
\left(L_{c c}\right)\end{array}$ & $\begin{array}{l}\text { maxloss due to } \\
\text { missing a pair of } \\
\text { factorial points } \\
\left(L_{f f}\right)\end{array}$ & $\begin{array}{l}\text { maxloss due to } \\
\text { missing a pair of } \\
\text { whole-plot axial } \\
\text { points }\left(L_{\alpha \alpha}\right)\end{array}$ & $\begin{array}{l}\text { maxloss due to } \\
\text { missing a pair of } \\
\text { subplot axial } \\
\text { points }\left(L_{\beta \beta}\right)\end{array}$ & $\begin{array}{l}\text { maxloss due to } \\
\text { missing one factorial } \\
\text { and one subplot axial } \\
\text { points }\left(L_{f \beta}\right)\end{array}$ & $\begin{array}{l}\text { Overall maxloss } \\
\text { due to missing } \\
\text { pairs of } \\
\text { observations }\end{array}$ \\
\hline 1.0 & 0.0183 & 0.9994 & 0.0265 & 0.7383 & 0.9861 & 0.9994 \\
\hline 1.20 & 0.0207 & 0.9991 & 0.0281 & 0.7611 & 0.9821 & 0.9991 \\
\hline 1.50 & 0.0255 & 0.9984 & 0.0298 & 0.7958 & 0.9767 & 0.9984 \\
\hline 2.0 & 0.0343 & 0.9966 & 0.0317 & 0.8496 & 0.9007 & 0.9966 \\
\hline 2.5 & 0.0342 & 0.9945 & 0.0330 & 0.8944 & 0.9675 & 0.9945 \\
\hline 3.0 & 0.0278 & 0.9931 & 0.0338 & 0.9269 & 0.9676 & 0.9931 \\
\hline 3.5 & 0.0226 & 0.9921 & 0.0343 & 0.9490 & 0.9693 & 0.9921 \\
\hline 4.0 & 0.0194 & 0.9915 & 0.0346 & 0.9639 & 0.9717 & 0.9915 \\
\hline 5.995 & 0.0146 & $0.9893 * * *$ & 0.0352 & $0.9893 * * *$ & 0.9813 & $0.9893 * * *$ \\
\hline
\end{tabular}




\begin{tabular}{lllllll}
\hline 6.3 & 0.0143 & 0.9392 & 0.0352 & 0.9909 & 0.9824 & 0.9909 \\
\hline
\end{tabular}

***Minimaxloss due to two missing observations.

Table 7: Losses due to different pairs of missing observations for five-factor $\mathrm{D}(2,3)$ split-plot CCD with half replication of factorial and complete replication of axial parts; $n_{f}=16$ from half replicate of factorial part with highest-order interaction as defining contrast, $n_{\alpha}=16, n_{\beta}$ $=6, n_{c}=4, N_{w}=10$, and $N=42$.

\begin{tabular}{llllll}
\hline$\alpha$ & $\begin{array}{l}\text { maxloss due to } \\
\text { missing a pair of } \\
\text { center points }\left(L_{c c}\right)\end{array}$ & $\begin{array}{l}\text { maxloss due to } \\
\text { missing a pair of } \\
\text { factorial points } \\
\left(L_{f f}\right)\end{array}$ & $\begin{array}{l}\text { maxloss due to } \\
\text { missing a pair of } \\
\text { whole-plot axial } \\
\text { points }\left(L_{\alpha \alpha}\right)\end{array}$ & $\begin{array}{l}\text { maxloss due to } \\
\text { missing a pair of } \\
\text { subplot axial points } \\
\left(L_{\beta \beta}\right)\end{array}$ & $\begin{array}{l}\text { maxloss due to } \\
\text { missing one factorial } \\
\text { and one subplot axial } \\
\text { points }\left(L_{f \beta}\right)\end{array}$ \\
\hline 1.0 & 0.0627 & 0.9985 & 0.1094 & 0.7173 & 0.9853 \\
1.20 & 0.0731 & 0.9974 & 0.1163 & 0.7381 & 0.9813 \\
1.50 & 0.0975 & 0.9949 & 0.1251 & 0.7748 & 0.9761 \\
2.0 & 0.1550 & 0.9886 & 0.1359 & 0.8314 & 0.9700 \\
2.5 & 0.1541 & 0.9809 & 0.1444 & 0.8821 & 0.9678 \\
3.0 & 0.1082 & 0.9739 & 0.1506 & 0.9196 & 0.9688 \\
3.5 & 0.0786 & 0.9677 & 0.1545 & 0.9442 & 0.9710 \\
4.043 & 0.0619 & $0.9617 * * *$ & 0.1573 & $0.9617 * * *$ & 0.9738 \\
4.5 & 0.0541 & 0.9574 & 0.1590 & 0.9717 & 0.9762 \\
5.0 & 0.0488 & 0.9533 & 0.1604 & 0.9793 & 0.9787 \\
\hline *** & & & & & \\
\hline
\end{tabular}

***Minimaxloss due to two missing observations.

Table 8: Losses due to different pairs of missing observations for six-factor $\mathrm{D}(2,4)$ split-plot CCD with half replication of factorial and complete replication of axial parts; $n_{f}=32$ from half replicate of factorial part with highest-order interaction as defining contrast, $n_{\alpha}=32, n_{\beta}$ $=8, n_{c}=8, N_{w}=10$, and $N=80$.

\begin{tabular}{llllll}
\hline$\alpha$ & $\begin{array}{l}\text { maxloss due to } \\
\text { missing a pair of } \\
\text { center points } \\
\left(L_{c c}\right)\end{array}$ & $\begin{array}{l}\text { maxloss due to } \\
\text { missing a pair of } \\
\text { factorial points } \\
\left(L_{f f}\right)\end{array}$ & $\begin{array}{l}\text { maxloss due to } \\
\text { missing a pair of } \\
\text { whole-plot axial } \\
\text { points }\left(L_{\alpha \alpha}\right)\end{array}$ & $\begin{array}{l}\text { maxloss due to missing } \\
\text { a pair of subplot axial } \\
\text { points }\left(L_{\beta \beta}\right)\end{array}$ & $\begin{array}{l}\text { maxloss due to missing } \\
\text { one factorial and one } \\
\text { subplot axial points } \\
\left(L_{f \beta}\right)\end{array}$ \\
\hline 1.0 & 0.0145 & 0.8711 & 0.0235 & 0.7089 & 0.8070 \\
1.20 & 0.0163 & 0.8681 & 0.0250 & 0.7226 & 0.8100 \\
1.50 & 0.0201 & 0.8629 & 0.0270 & 0.7451 & 0.8153 \\
2.0 & 0.0300 & 0.8532 & 0.0294 & 0.7851 & 0.8261 \\
2.5 & 0.0356 & 0.8427 & 0.0310 & 0.8261 & 0.8396 \\
2.665 & 0.0343 & $0.8394 * * *$ & 0.0315 & $0.8394 * * * 8643$ & 0.8446 \\
3.0 & 0.0287 & 0.8329 & 0.0322 & 0.8951 & 0.8550 \\
3.5 & 0.0208 & 0.8240 & 0.0331 & 0.9188 & 0.8835 \\
4.0 & 0.0159 & 0.8158 & 0.0336 & & \\
\hline
\end{tabular}

****Minimaxloss due to two missing observations.

Table 9: Losses due to different pairs of missing observations for 6 -factor $\mathrm{D}(3,3)$ split-plot CCD with half replicationof factorial and complete replication of axial parts; $n_{f}=32$ from half replicate of factorial part with highest-order interaction as defining contrast, $n_{\alpha}=24, n_{\beta}=6, n_{c}=4$, $N_{w}=16$, and $N=66$.

\begin{tabular}{llllll}
\hline$\alpha$ & $\begin{array}{l}\text { maxloss due to } \\
\text { missing a pair of } \\
\text { center points }\left(L_{c c}\right)\end{array}$ & $\begin{array}{l}\text { maxloss due to } \\
\text { missing a pair of } \\
\text { factorial points } \\
\left(L_{f f}\right)\end{array}$ & $\begin{array}{l}\text { maxloss due to } \\
\text { missing a pair of } \\
\text { whole-plot axial } \\
\text { points }\left(L_{\alpha \alpha}\right)\end{array}$ & $\begin{array}{l}\text { maxloss due to } \\
\text { missing a pair of } \\
\text { subplot axial } \\
\text { points }\left(L_{\beta \beta}\right)\end{array}$ & $\begin{array}{l}\text { maxloss due to missing } \\
\text { one factorial and one } \\
\text { subplot axial points } \\
\left(L_{f \beta}\right)\end{array}$ \\
\hline 1.0 & 0.0477 & 0.8653 & 0.0988 & 0.6824 & 0.7951 \\
1.50 & 0.0713 & 0.8558 & 0.1116 & 0.7210 & 0.8033 \\
2.0 & 0.1247 & 0.8447 & 0.1223 & 0.7619 & 0.8137 \\
2.5 & 0.1659 & 0.8328 & 0.1313 & 0.8054 & 0.8274 \\
2.739 & 0.1470 & $0.8278^{* * *}$ & 0.1355 & $0.8278 * * *$ & 0.8359 \\
3.0 & 0.1149 & 0.8230 & 0.1395 & 0.8504 & 0.8455 \\
3.5 & 0.0705 & 0.8148 & 0.1455 & 0.8853 & 0.8624 \\
4.0 & 0.0492 & 0.8074 & 0.1497 & 0.9115 & 0.8773 \\
\hline
\end{tabular}

**Minimaxloss due to two missing observations.

Table 10: Losses due to different pairs of missing observations for six-factor $\mathrm{D}(1,5)$ split-plot CCD with halfreplicationof factorial and complete replication of axial parts; $n_{f}=32$ from half replicate of factorial part with highest-order interaction as defining contrast, $n_{\alpha}=32, n_{f}$ $=10, n_{c}=16, N_{w}=6$, and $N=90$.

\begin{tabular}{llllll}
\hline$\alpha$ & $\begin{array}{l}\text { maxloss due to } \\
\text { missing a pair of } \\
\text { center points } \\
\left(L_{c c}\right)\end{array}$ & $\begin{array}{l}\text { maxloss due to } \\
\text { missing a pair of } \\
\text { factorial points } \\
\left(L_{f f}\right)\end{array}$ & $\begin{array}{l}\text { maxloss due to } \\
\text { missing a pair of } \\
\text { whole-plot axial } \\
\text { points }\left(L_{\alpha \alpha}\right)\end{array}$ & $\begin{array}{l}\text { maxloss due to } \\
\text { missing a pair of } \\
\text { subplot axial points } \\
\left(L_{\beta \beta}\right)\end{array}$ & $\begin{array}{l}\text { maxloss due to missing } \\
\text { one factorial and one } \\
\text { subplot axial points } \\
\left(L_{f \beta}\right)\end{array}$ \\
\hline 1.0 & 0.0043 & 0.8860 & 0.0062 & 0.7241 & 0.8218 \\
1.50 & 0.0056 & 0.8779 & 0.0070 & 0.7592 & 0.8292 \\
2.0 & 0.0074 & 0.8679 & 0.0074 & 0.7984 & 0.8390 \\
2.5 & 0.0083 & 0.8571 & 0.0077 & 0.8374 & 0.8510 \\
2.706 & 0.0080 & $0.8526 * * *$ & 0.0077 & $0.8526 * * *$ & 0.8564 \\
3.0 & 0.0073 & 0.8463 & 0.0078 & 0.8724 & 0.8642 \\
3.5 & 0.0060 & 0.8362 & 0.0080 & 0.9008 & 0.8771 \\
4.0 & 0.0050 & 0.8269 & 0.0080 & 0.9230 & 0.8893 \\
\hline
\end{tabular}


Loss curves due to a pair of missing observations for split-plot CCDs with half replicate of factorial part and single replicate of axial part
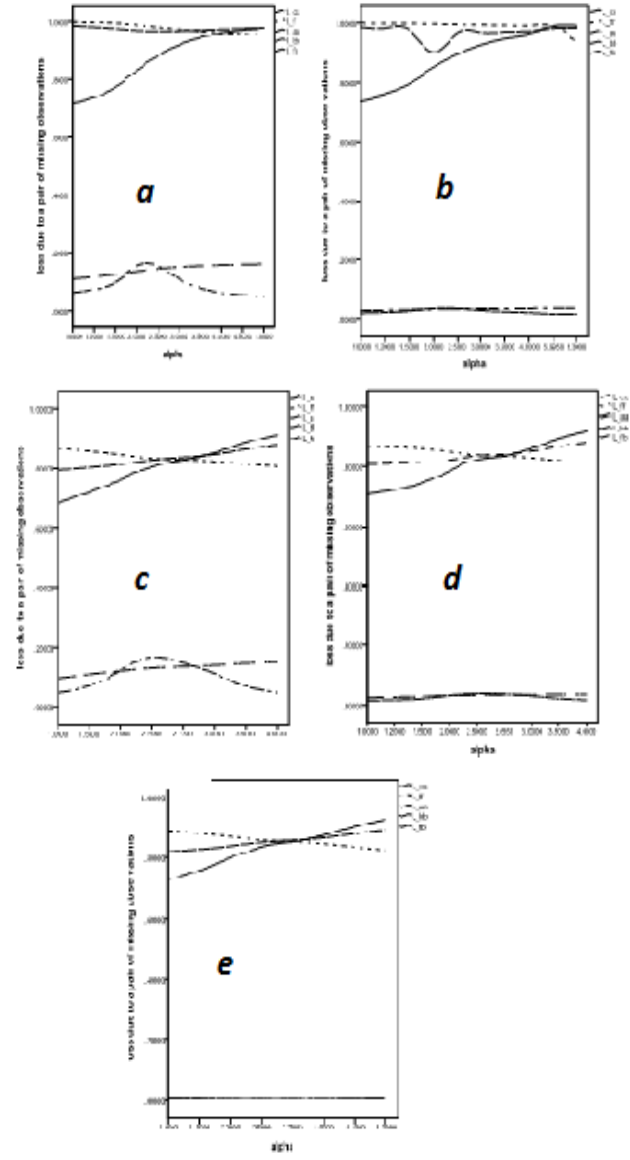

Figures 2: Loss curves due to a pair of missing observations for (a) $[\mathrm{D}(1,4)](\mathrm{b})[\mathrm{D}(2,3)](\mathrm{c})[\mathrm{D}(2,4)]$ (d) $[\mathrm{D}(3,3)]$ and (e) $[\mathrm{D}(1,5)]$ with half replicate of factorial part and complete replicate of axial part $(1 / 2 \mathrm{~F}+\mathrm{A})$.

Conclusions: This study has established the robustness potentials of split-plot central composite designs when different pairs of observations were missing. The study has identified the most influential points of these designs to be the factorial $(f)$ and the subplot axial $(\beta)$ points while the less influential ones are the whole-plot axial and the center points. The study revealed a gradual decrease in the losses, $L_{f f}$, due to missing pairs of factorial points and an increase in the losses, $L_{\beta \beta}$, due to missing pairs of subplot axial points as the axial point distance of these designs increases from the design center. Thus design efficiency can be increased considerably by avoiding less influential points from the full split-plot CCD.

\section{REFERENCES}

Akhtar, M.; Prescott, P (1986). Response Surface Designs Robust to Missing Observations. Communications in Statistics and Simulations 15(2): 345-363.

Akhtar M. (2001). Five-factor central composite design robust to a pair of Missing observations. Journal of Research (Science), Bahauddin Zakariya University, Multan, Pakistan, Vol.12, No.2: 105-115.

Akram M. (2002). Central Composite Designs Robust to Three Missing Observations. Unpublished Ph.D. dissertation, the Islamia University, Bahawalpur, Pakistan.

Angela U. C; Yisa Y; Bamiduro TA; Amahia G. N. (2013). Robustness of split-plot CCDs in the presence of a single missing observation. Pacific Journal of Science and Technology, Vol. 14, No. 2: 194-211.

Letsinger, J. D; Myers, RH; Lentner, M (1996). Response Surface Methods for Bi-randomization Structures. Journal of Quality Technology 28:381-397.

Myers, R. H; Montgomery, DC; Christine, MA (2009). Response Surface Methodology, Process And Product Optimization Using Designed Experiments. Wiley, New York, NY.

Kowalski, SM; Geoffrey, GV; Douglas, CM; Connie, MB (2006). Modifying central composite designs to model the process mean and variance when there are hard-to-change factors. Journal of Royal Statistical Society, series C. Vol. 55 no. 5: 615630.

Vining, GG; Kowalski, SM; Montgomery, DC (2005). Response Surface Designs Within a Split-Plot Structure. Journal of Quality Technology 37: 115129. 